\title{
Determinantes de exposición a material particulado en el área textil
}

\section{(Determinants of exposure to particulate matter in textiles)}

\author{
Carlos Rosales ${ }^{1}$, Jorge Viteri², Nadya Rivera ${ }^{1}$, Raúl Comas ${ }^{3}$
}

\begin{abstract}
Resumen:
El objetivo de la investigación fue determinar la exposición a material particulado en una empresa textil en la línea de producción de blanquería (fabricación de sábanas y edredones). Las mediciones se efectuaron para la fracción respirable $2,5 \mu \mathrm{g} / \mathrm{m}^{3}$ mediante el uso de un equipo de lectura directa. El estudio determinó que la operación con las máquinas overlock es donde se genera mayor cantidad de material particulado, con un valor máximo de $244 \mu \mathrm{g} / \mathrm{m}^{3}$, y un mínimo en la máquina dobladora con un valor de $9 \mu \mathrm{g} / \mathrm{m}^{3}$. Durante la investigación se conoció la percepción de los empleados respecto a su trabajo.
\end{abstract}

Palabras clave: organización del trabajo; fracción respirable; límite de exposición

\begin{abstract}
:
The objective of the research was to determine the exposure to particulate material in the textil area of a manufacturing company of linens and duvets, where it is carried out measurements of particulate material in respirable fraction $2.5 \mu \mathrm{g} / \mathrm{m} 3$. The same were made through the use of the equipment of direct reading and the results are compared with the value set limit. The study found that in the operation with the overlock machines is where there is a greater amount of particulate matter getting the maximum value in the entire production plant of $244 \mu \mathrm{g} / \mathrm{m}^{3}$, and a lower value machine in the bender with a value of $9 \mu \mathrm{g} / \mathrm{m}^{3}$ During the course of the investigation, it wanted to know the perception of the employees, to do this a survey was administered in a manner that takes first-hand the opinion with regard to the work. Among the results is that the staff is aware that there is particulate matter and if you have had respiratory complaints, indicate that the organization presents a great deal of commitment to the security and they also are involved in the tasks.
\end{abstract}

Keywords: work organization; respirable fraction; Exposure Limit

\section{Introducción}

En la actualidad los cambios que se han generado en materia de seguridad y salud ocupacional para disminuir los niveles de accidentes y precautelar el bienestar de los trabajadores (Gallego C., Paeres R., \& Gómez, 2010), son importantes. En el Ecuador se han generado leyes, acuerdos, decretos en función de la seguridad y salud ocupacional como son: la Constitución de la Republica

\footnotetext{
${ }_{1}^{1}$ Universidad Tecnológica Equinoccial, Quito - Ecuador (\{carlos.rosales, nivera\}@ute.edu.ec)

${ }^{2}$ Universidad Central del Ecuador, Quito - Ecuador (jrviteri@uce.edu.ec)

${ }^{3}$ Universidad de Sancti Spíritus, Sancti Spíritus - Cuba (raul@uniss.edu.cu)
} 
en sus artículos $33^{4}$ y $326^{5}$, el Instrumento Andino de Seguridad y Salud en el trabajo (Decisión 584), Código del Trabajo del Ecuador, Reglamento del Seguro General de Riesgos del Trabajo (Resolución C.D. 390), Reglamento de Seguridad y Salud de los Trabajadores y Mejoramiento del Medio Ambiente de Trabajo (Decreto Ejecutivo 2393), Sistema de Auditorias de Riesgos del Trabajo (SART Resolución C.D. 333). Estas normativas conllevan a tomar en cuenta la seguridad y prevención de riesgos tanto en los sitios de trabajo como en las personas.

La cultura de la prevención de riesgos es fundamentada en el compromiso y la participación (OISS, 2009). En el país, hace más de dos décadas, existe un marco legal, descrito anteriormente, que presenta la cultura de la prevención como el eje, objetivo y medio fundamental para conseguir una mejora efectiva de las condiciones de vida y de trabajo de la población laboralmente activa. Sin embargo, se puede decir que aún no se ha iniciado este proceso de manera consciente y no se ha logrado obtener resultados altamente satisfactorios en su aplicación en los diferentes medios de producción en el país. Es así como en múltiples sectores se muestra: un interés por la obligación y el cumplimiento legal en materia de seguridad, generar una cultura, reducir la siniestralidad y enfermedades laborales.

A pesar de la existencia de una base legal, que fomenta el desarrollo de la cultura de prevención, las empresas están trabajan en la concientización en seguridad y salud ocupacional y no han alcanzado una madurez apropiada. Las actividades laborales conllevan a que puedan haber eventos nocivos o no deseados que afectan la salud humana, ya sea por las condiciones en las que realiza las actividades o por el entorno laboral (Creus \& Magnosio, 2011). Los trabajadores y empleadores observan que no se cumplen las exigencias normativas y los sistemas de control no son suficientes para la implantación adecuada de un sistema de prevención, como resultado se tiene una alta accidentalidad.

Un aspecto importante es el relacionado con la Responsabilidad Social Empresarial (RSE). El empresario asume un compromiso voluntario de como contribuir al desarrollo social sustentable desde el punto de vista sistémico. La RSE es un instrumento integrador que considera diversos elementos y relaciones de la estructura de una organización para: la identificación de necesidades, determinación de estrategias, la selección, aplicación y evaluación de los resultados obtenidos; esto que genera un modelo de gestión que permite a la empresa incorporar dentro de

\footnotetext{
4 "El trabajo es un deber y un derecho social, y un derecho económico, fuente de realización personal y base de la economía. El estado garantizará a las personas trabajadores, el pleno respeto a su dignidad, una vida decorosa, remuneraciones y retribuciones justas y el desempeño de un trabajo saludable y libremente escogido y aceptado".

${ }^{5}$ El derecho al trabajo se sustenta en los siguientes principios:

5. Toda persona tendrá derecho a desarrollar sus labores en un ambiente adecuado y propicio, que garantice su salud, integridad, seguridad, higiene y bienestar.

6. Toda persona rehabilitada después de un accidente de trabajo o enfermedad, tendrá derecho a ser reintegrada al trabajo y a mantener la relación laboral, de acuerdo con la ley.
} 
su planificación estratégica herramientas para responder a las necesidades del entorno y sus stakeholders o grupos de interés. Las empresas deben hacerlos partícipes en los procesos de toma de decisiones institucionales para alcanzar la excelencia. (Viteri \& Jácome, La Responsabilidad Social como Modelo de Gestión Empresarial, 2013)

La industria textil del Ecuador, en el tiempo, ha generado empleo, tanto directo como indirecto, llegando a ser uno de los sectores manufactureros que más mano de obra emplea, después del sector alimentario. En el país hay alrededor de 3500 negocios, entre grandes, pequeños y formales, dedicado a la fabricación textil, con una producción estimada de 770 MMUSD para el 2013. (AITE, 2014)

El sector industrial textil necesita cotidianamente la evaluación de los determinantes de material particulado, ya que en las organizaciones, si bien se conoce que existe contaminación en el ambiente laboral, se evalúa poco el nivel de exposición al que son sometidos los trabajadores.

En la planta objeto de estudio las condiciones de trabajo son adecuadas y los trabajadores cuentan con medio de protección, pero la seguridad y salud ocupacional se lleva deficientemente, no se cuenta con los registros de la actividad, ni de las acciones de capacitación que se realizan. Para la investigación se tomaron muestras de cada sitio de trabajo y se determinó la cantidad de material particulado al que se encuentran expuestos los trabajadores, con esto se logra conocer en qué operación o máquina se genera mayor cantidad de este material.

Para la realización del estudio se evaluó la organización del trabajo, teniendo en cuenta:

- Jornada de trabajo.

- Tipo de cargo.

- Rutina de trabajo.

También se evaluaron las condiciones de trabajo:

- El mantenimiento (limpieza).

- Conocimientos sobre seguridad e higiene del trabajo.

- Características de la tarea.

Por todo lo anterior planteado, el objetivo de la investigación es determinar la exposición a material particulado en una empresa textil en la línea de producción de blanquería (fabricación de sábanas y edredones). 


\section{Materiales y métodos}

Se realizó un estudio de corte transversal según los lineamientos de la investigación descriptiva y correlacional de tipo cuantitativo con rasgos de cualitativo. Se utilizó también el análisis documental con un estudio de los principales documentos rectores de la empresa objeto de estudio.

El sistema de variables que orientó la investigación se muestra en la figura 1. Las variables consideradas son: las normas básicas de seguridad, herramientas en el proceso de fabricación, ambiente de trabajo y niveles de concentración de material particulado.

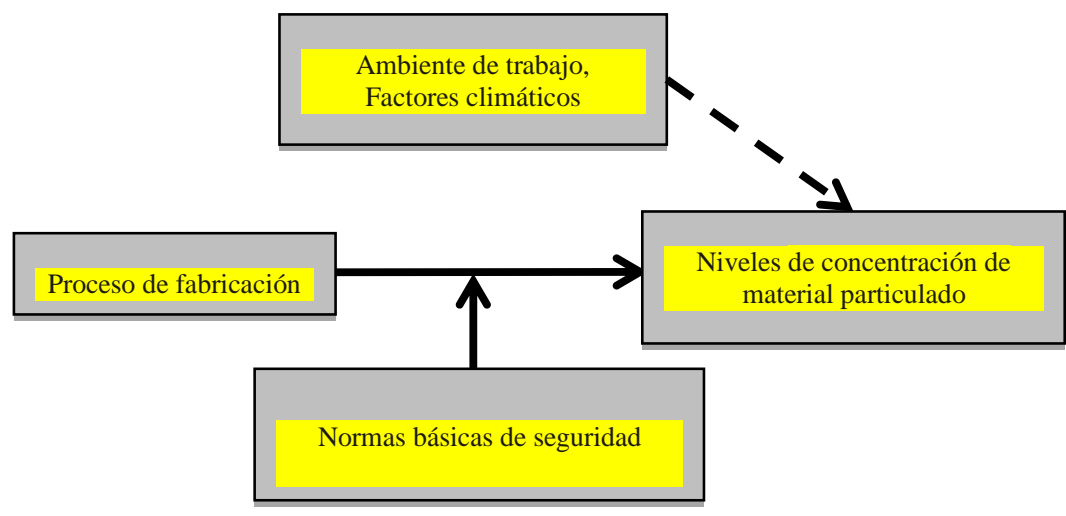

Figura 1. Sistema de variables

Para la aplicación de la encuesta de recolección de datos, al personal que labora en el centro de trabajo y por el número de trabajadores en la planta, se determinó aplicarla a toda la población y realizar las mediciones en el 100\% de la planta de producción textil.

\section{Métodos, técnicas e instrumentos de la Investigación}

Para obtener los resultados se utilizó el método descrito en la nota técnica de prevención del Instituto Nacional de Seguridad e Higiene del trabajo (INSTH) NTP 808 (Marti Veciana, Antonio; Guardino Solá, Xavier, 2008), y la estrategia de medición según la norma europea UNE-EN 689 (CENELEC, 1995) la cual indica:

- Definir el tipo de contaminante que existe.

- Seleccionar la muestra para la medición de exposición.

- Toma de muestra con un instrumento de medición.

En caso de la medición ser de corta duración, como en el caso de estudio, los requisitos a cumplir de acuerdo a la nota técnica del INSHT NTP 808 se muestran en la tabla 1 (Marti Veciana, Antonio; Guardino Solá, Xavier, 2008). 
Tabla 1. Requisitos de medida de material particulado

\begin{tabular}{|c|c|}
\hline $\begin{array}{c}\text { OBJETO DE LA } \\
\text { MEDIDA }\end{array}$ & REQUISITOS DEL PROCEDIMIENTO DE MEDIDA \\
\hline $\begin{array}{l}\text { Evaluación inicial de la } \\
\text { concentración ponderada } \\
\text { en el tiempo }\end{array}$ & $\begin{array}{ll} & \text { Selectividad adecuada al agente químico; } \\
\text { - } & \text { tiempo de muestreo menor o igual al período de referencia del VL; } \\
\text { - } \quad \text { un intervalo de medida que incluya el valor límite; } \\
\end{array}$ \\
\hline $\begin{array}{l}\text { Evaluación inicial de la } \\
\text { concentración con el } \\
\text { tiempo o en el espacio }\end{array}$ & $\begin{array}{l}\text { - } \quad \text { selectividad adecuada al agente químico; } \\
\text { - } \quad \text { un tiempo de muestreo corto ( } \leq 5 \mathrm{~min} \text { ó } \leq 15 \mathrm{~min} \text {, según sean variaciones } \\
\text { - un intervalo de medida el que se ajuste al objeto de la medición; } \\
\text { - una incertidumbre expandida que se ajuste a la finalidad de la medición. }\end{array}$ \\
\hline $\begin{array}{l}\text { Mediciones de } \\
\text { comparación con los } \\
\text { valores límite y } \\
\text { mediciones periódicas }\end{array}$ & $\begin{array}{l}\text { - } \quad \text { no ambigüedad, en el intervalo de medida específico y en los resultados de } \\
\text { - } \quad \text { la concentración del agente químico que se está midiendo; } \\
\text { - } \quad \text { cualquier interferencia; } \\
\text { igual al período de referencia del VL; } \\
\text { - } \quad \text { intervalo de medida que cubra de } 0,1 \text { a } 2 \text { veces el VL para medidas a largo } \\
\text { plazo y de } 0,2 \text { a } 2 \text { veces el VL para las de corta duración; } \\
\text { - } \quad \text { los requisitos de la incertidumbre expandida que figuran en Tabla 2; } \\
\text { - el transporte y el almacenamiento de las muestras cuando sea apropiado, } \\
\text { debe llevar a cabo de modo que se mantenga la integridad física y } \\
\text { química; } \\
\text { - los efectos de las condiciones ambientales sobre el funcionamiento del } \\
\text { método debe ser ensayadas en condiciones de laboratorio, mientras que } \\
\text { los demás (ambigüedad, selectividad, incertidumbre, etc.) en el lugar de } \\
\text { trabajo; } \\
\text { - los procedimientos de medida deben ser redactados según la Norma ISO } \\
\text { 78-2, conteniendo toda la información necesaria para llevar a cabo el } \\
\text { procedimiento de medida (ver NTP 547:2000). }\end{array}$ \\
\hline
\end{tabular}

Fuente: (Marti Veciana, Antonio; Guardino Solá, Xavier, 2008)

\section{Criterios de valoración}

El procedimiento para las mediciones de material particulado es el establecido por la NIPO 21106-050-8 (INSHT, 2006). Se toma una muestra de la fracción inhalable de materia particulada, índicador requerido por el reglamento de seguridad y salud de los trabajadores y mejoramiento del medio ambiente (IESS, 1986).

Posteriormente los datos obtenidos serán comparados con los valores Límites Máximos Permisibles (TLV) de exposición, que para el caso de estudio se comparará con el valor límite del algodón.

La toma de muestra se realiza durante el intervalo de costura, en la línea de producción de blanquería, que varía de acuerdo a la prenda que se confecciona.

\section{Instrumento de medición}

Considerando las características y existencia en el mercado y la lectura inmediata se escoge el equipo de lectura directa EVM de la empresa 3M. El equipo EVM es un equipo compacto, de fácil manejo, con capacidad para monitorear simultáneamente y en tiempo real, varios parámetros de calidad del aire y partículas, sus características se muestran en la tabla 2. 
Tabla 2. Especificaciones del instrumento de medición

\begin{tabular}{|c|c|}
\hline \multicolumn{2}{|c|}{ ESPECIFICACIONES } \\
\hline $\begin{array}{ll}\text { Tipo: } & \text { EVM } \\
\text { № de serie: } & \text { ENK050002 } \\
\text { Firmware: } & \text { R.10 } \\
\text { Ultima recalibración: } 29 / 03 / 2013\end{array}$ & Condiciones: $\begin{aligned} & 0-+50^{\circ} \mathrm{C} /-20-+60^{\circ} \mathrm{C} \\
& 10-90 \% \mathrm{HR} / 0-95 \% \mathrm{HR} \\
& 65 \mathrm{kPa}-108 \mathrm{kPa}\end{aligned}$ \\
\hline $\begin{array}{l}\text { Características: Partículas, Humedad relativa } \\
\text { Temperatura }\end{array}$ & \\
\hline $\begin{array}{l}\text { Sensor de partículas: } \\
\text { Tamaño de partícula: } 0,1 \mu \mathrm{m} \text { a } 10 \mu \mathrm{m} \text { (puede detectar } \\
\text { partículas sobre las } 100 \mu \mathrm{m}) \\
\text { Selector mecánico de partículas } \\
\text { Selector de PM } 2,5-\mathrm{PM} 4-\mathrm{PM} 10 \text { o PM }(1,67 \mathrm{~L} / \mathrm{min}) \\
\text { Unidades. } \mathrm{mg} / \mathrm{m}^{3}-\mu \mathrm{g} / \mathrm{m}^{3} \\
\text { Muestreo gravimétrico: flow: } \pm 5 \% \\
\quad \text { back-pressure cassette gravimétrico } \\
\quad>5 \mathrm{kPa} \\
\quad>20 \text { inch of water } 1,67 \mathrm{lpm} \\
\text { Sensor de humedad relativa } \\
\text { Sensor capacitivo Rango: } 0-100 \% \text { Error } 0.1 \\
\text { Sensor de temperatura Rango: } 0-60 \stackrel{\circ}{ } \mathrm{C} \text { Error: } 0,1\end{array}$ & $\begin{array}{l}\text { Sensor: CO } \\
\text { Sensor: CO2 } \\
\text { Sensor: PID Voc }\end{array}$ \\
\hline
\end{tabular}

\section{Instrumento de recolección de datos}

Para la recolección de datos y conocer la percepción de los empleados se realizó una encuesta a los treinta operadores de la planta de producción, validándola por el método Delphi. Se realizaron cuatro rondas hasta llegar a un consenso entre los expertos que valoraron la encuesta.

\section{Resultados}

Previo a la obtención de resultados se debe indicar que la organización trabaja en una sola jornada de 7:00 de la mañana hasta las 15:30 de las cuales son 8 horas laborables y 30 minutos de almuerzo. Para realizar las mediciones de material particulado en la planta de confección es necesario indicar que se encuentra dividido en tres grupos, que tienen dos máquinas de overlock, en la zona de confección de sábanas, una máquina de costura recta, una máquina para dobleces y una mesa para el empacado. Luego de los grupos de confección está la zona de telas para corte, y al otro lado se tiene la zona de fabricación de edredones y bordados.

Para la toma de muestra se realizó durante la labor de costura utilizando un ciclo completo que en este caso es de duración corta es decir menor o igual a 5 minutos, de acuerdo a la norma citada en la tabla 1. Los sitios donde se tomaron las muestras para las mediciones del material particulado fueron establecidos y se muestran en la tabla 3. 
Tabla 3. Listado de toma de muestra en los sitios de planta.

\begin{tabular}{|c|c|c|}
\hline Muestra N.- & Grupo & Sitio/máquina \\
\hline 1 & 1 & Mesa de corte de hilos/empacado \\
\hline 2 & 1 & Máquina overlock 1 \\
\hline 3 & 1 & Máquina de costura recta \\
\hline 4 & 1 & Máquina dobladora \\
\hline 5 & 1 & Máquina overlock 2 \\
\hline 6 & 2 & Mesa de corte de hilos/empacado \\
\hline 7 & 2 & Máquina overlock 1 \\
\hline 8 & 2 & Máquina de costura recta \\
\hline 9 & 2 & Máquina dobladora \\
\hline 10 & 2 & Máquina overlock2 \\
\hline 11 & 3 & Mesa de corte de hilos/empacado \\
\hline 12 & 3 & Máquina de costura recta \\
\hline 13 & 3 & Máquina overlock1 \\
\hline 14 & 3 & Máquina overlock2 \\
\hline 15 & 3 & Máquina dobladora \\
\hline 16 & Acolchado & Acolchadora automática \\
\hline 17 & Acolchado & Confección edredones acolchados \\
\hline 18 & Bordado & Bordadoras automáticas \\
\hline 19 & Pasillo & Frontal \\
\hline 20 & Pasillo & Posterior \\
\hline 21 & Corte & Mesa de corte de telas \\
\hline
\end{tabular}

En la tabla 4 se presenta un resumen general de las mediciones tanto en valores máximos como en promedio y el valor permisible para partículas o polvo de algodón, ya que la composición de la tela a trabajar es de un $60 \%$ de algodón y $40 \%$ de material poliéster. En la figura 2 se presenta un esquema de la distribución de planta donde se tomaron las mediciones

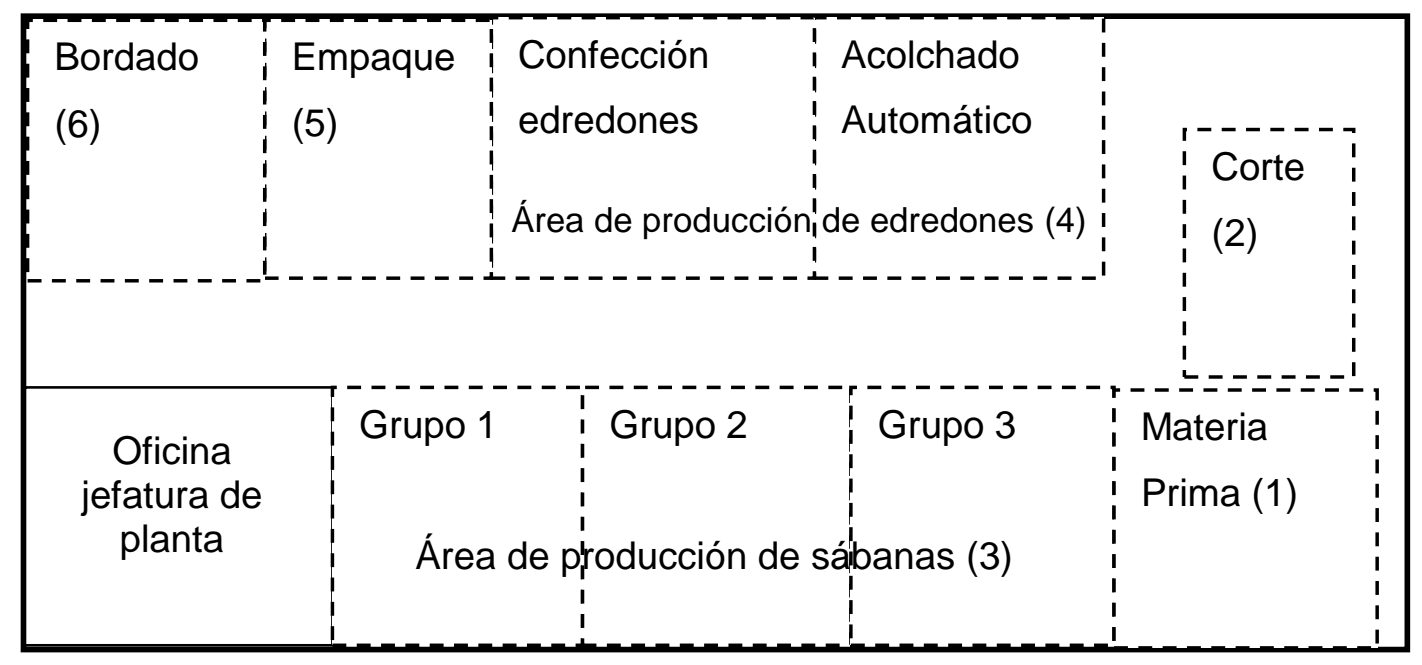

Figura 2. Distribución de áreas en planta 
Tabla 4. Resumen de los datos obtenidos en la planta de confección

\begin{tabular}{|c|c|c|c|c|c|c|}
\hline Instrumento & Tipo: EVM & Marca: Quest & \multicolumn{2}{|c|}{ Nombre: ENK050002 } & \multicolumn{2}{|c|}{$\begin{array}{l}\text { Número de serie: } \\
\text { ENK050002 }\end{array}$} \\
\hline & Calibración & \multicolumn{5}{|c|}{ 26-06-2014 06:23:00 } \\
\hline $\begin{array}{l}\text { GRUPO DE } \\
\text { TRABAJO }\end{array}$ & \multicolumn{2}{|c|}{ PUESTO } & MÁXIMO & \multicolumn{2}{|c|}{ PROMEDIO } & $\begin{array}{l}\text { TLV`s (polvo de } \\
\text { algodón) }\end{array}$ \\
\hline Grupo 1 & \multicolumn{2}{|c|}{ Mesa de corte de hilos/empacado } & $31 \mu \mathrm{g} / \mathrm{m}^{3}$ & \multicolumn{2}{|c|}{$17 \mu \mathrm{g} / \mathrm{m}^{3}$} & $100 \mu \mathrm{g} / \mathrm{m}^{3}$ \\
\hline Grupo 1 & \multicolumn{2}{|c|}{ Máquina overlock 1} & $29 \mu \mathrm{g} / \mathrm{m}^{3}$ & \multicolumn{2}{|c|}{$14 \mu \mathrm{g} / \mathrm{m}^{3}$} & $100 \mu \mathrm{g} / \mathrm{m}^{3}$ \\
\hline Grupo 1 & \multicolumn{2}{|c|}{ Máquina de costura recta } & $27 \mu \mathrm{g} / \mathrm{m}^{3}$ & \multicolumn{2}{|c|}{$16 \mu \mathrm{g} / \mathrm{m}^{3}$} & $100 \mu \mathrm{g} / \mathrm{m}^{3}$ \\
\hline Grupo 1 & \multicolumn{2}{|c|}{ Máquina dobladora } & $33 \mu \mathrm{g} / \mathrm{m}^{3}$ & \multicolumn{2}{|c|}{$17 \mu \mathrm{g} / \mathrm{m}^{3}$} & $100 \mu \mathrm{g} / \mathrm{m}^{3}$ \\
\hline Grupo 1 & \multicolumn{2}{|c|}{ Máquina overlock 2} & $29 \mu \mathrm{g} / \mathrm{m}^{3}$ & \multicolumn{2}{|c|}{$18 \mu \mathrm{g} / \mathrm{m}^{3}$} & $100 \mu \mathrm{g} / \mathrm{m}^{3}$ \\
\hline Grupo 2 & \multicolumn{2}{|c|}{ Mesa de corte de hilos/empacado } & $57 \mu \mathrm{g} / \mathrm{m}^{3}$ & \multicolumn{2}{|c|}{$23 \mu \mathrm{g} / \mathrm{m}^{3}$} & $100 \mu \mathrm{g} / \mathrm{m}^{3}$ \\
\hline Grupo 2 & \multicolumn{2}{|c|}{ Máquina overlock 1} & $219 \mu \mathrm{g} / \mathrm{m}^{3}$ & \multicolumn{2}{|c|}{$76 \mu \mathrm{g} / \mathrm{m}^{3}$} & $100 \mu \mathrm{g} / \mathrm{m}^{3}$ \\
\hline Grupo 2 & \multicolumn{2}{|c|}{ Máquina de costura recta } & $85 \mu \mathrm{g} / \mathrm{m}^{3}$ & \multicolumn{2}{|c|}{$56 \mu \mathrm{g} / \mathrm{m}^{3}$} & $100 \mu \mathrm{g} / \mathrm{m}^{3}$ \\
\hline Grupo 2 & \multicolumn{2}{|c|}{ Máquina dobladora } & $95 \mu \mathrm{g} / \mathrm{m}^{3}$ & \multicolumn{2}{|c|}{$63 \mu \mathrm{g} / \mathrm{m}^{3}$} & $100 \mu \mathrm{g} / \mathrm{m}^{3}$ \\
\hline Grupo 2 & \multicolumn{2}{|c|}{ Máquina overlock 2} & $244 \mu \mathrm{g} / \mathrm{m}^{3}$ & \multicolumn{2}{|c|}{$143 \mu \mathrm{g} / \mathrm{m}^{3}$} & $100 \mu \mathrm{g} / \mathrm{m}^{3}$ \\
\hline Grupo 3 & \multicolumn{2}{|c|}{ Mesa de corte de hilos/empacado } & $35 \mu \mathrm{g} / \mathrm{m}^{3}$ & \multicolumn{2}{|c|}{$17 \mu \mathrm{g} / \mathrm{m}^{3}$} & $100 \mu \mathrm{g} / \mathrm{m}^{3}$ \\
\hline Grupo 3 & \multicolumn{2}{|c|}{ Máquina de costura recta } & $45 \mu \mathrm{g} / \mathrm{m}^{3}$ & \multicolumn{2}{|c|}{$21 \mu \mathrm{g} / \mathrm{m}^{3}$} & $100 \mu \mathrm{g} / \mathrm{m}^{3}$ \\
\hline Grupo 3 & \multicolumn{2}{|c|}{ Máquina overlock 1} & $93 \mu \mathrm{g} / \mathrm{m}^{3}$ & \multicolumn{2}{|c|}{$72 \mu \mathrm{g} / \mathrm{m}^{3}$} & $100 \mu \mathrm{g} / \mathrm{m}^{3}$ \\
\hline Grupo 3 & \multicolumn{2}{|c|}{ Máquina overlock 2} & $126 \mu \mathrm{g} / \mathrm{m}^{3}$ & \multicolumn{2}{|c|}{$60 \mu \mathrm{g} / \mathrm{m}^{3}$} & $100 \mu \mathrm{g} / \mathrm{m}^{3}$ \\
\hline Grupo 3 & Máquina doblac & & $18 \mu \mathrm{g} / \mathrm{m}^{3}$ & $9 \mu \mathrm{g} / \mathrm{m}$ & & $100 \mu \mathrm{g} / \mathrm{m}^{3}$ \\
\hline & Acolchadora au & tica & $104 \mu \mathrm{g} / \mathrm{m}^{3}$ & $81 \mu \mathrm{g} / \mathrm{r}$ & & $100 \mu \mathrm{g} / \mathrm{m}^{3}$ \\
\hline & Confección edr & es acolchados & $106 \mu \mathrm{g} / \mathrm{m}^{3}$ & $82 \mu \mathrm{g} / \mathrm{r}$ & & $100 \mu \mathrm{g} / \mathrm{m}^{3}$ \\
\hline & Bordadoras aut & icas & $153 \mu \mathrm{g} / \mathrm{m}^{3}$ & $92 \mu \mathrm{g} / \mathrm{r}$ & & $100 \mu \mathrm{g} / \mathrm{m}^{3}$ \\
\hline & Frontal & & $148 \mu \mathrm{g} / \mathrm{m}^{3}$ & $92 \mu \mathrm{g} / \mathrm{r}$ & & $100 \mu \mathrm{g} / \mathrm{m}^{3}$ \\
\hline & Posterior & & $101 \mu \mathrm{g} / \mathrm{m}^{3}$ & $82 \mu \mathrm{g} / \mathrm{r}$ & & $100 \mu \mathrm{g} / \mathrm{m}^{3}$ \\
\hline & Mesa de corte $c$ & & $108 \mu \mathrm{g} / \mathrm{m}^{3}$ & $89 \mu \mathrm{g} / \mathrm{r}$ & & $100 \mu \mathrm{g} / \mathrm{m}^{3}$ \\
\hline & Niveles altos $\square$ & Niveles medios & Niveles : & $s \square$ & & \\
\hline
\end{tabular}

De la tabla anterior se observa que los puestos de costura con máquinas overlock y el área de fabricación de edredones generan la mayor cantidad de material particulado, tanto en los niveles máximos y promedio. Los pasillos en bordado presentan también de igual forma altos niveles que sobrepasan el nivel de $100 . \mu \mathrm{g} / \mathrm{m}^{3}$. En la figura 3 se observa los resultados de materia particulada en la máquina overlock 2 del grupo 2 que es la que presenta mayor número de partículas emitidas al ambiente. 


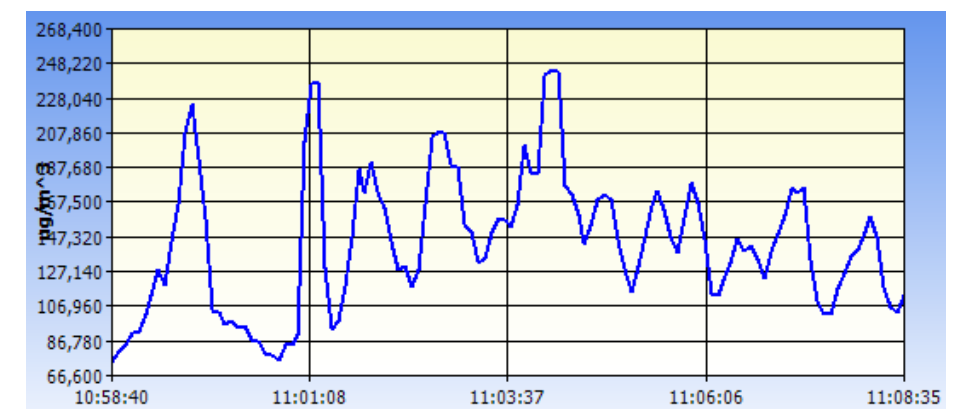

Figura 3. Resultado de muestreo en máquina overlock 2 grupo 2

Con la información obtenida de las encuestas se encontró que:

- La mayor cantidad de personas se encuentra entre 26 y 35 años y el promedio de edad es de 31.5 años.

- La gran mayoría por el tipo de actividad que realizan son mujeres (operadoras) y apenas 5 hombres. El $83 \%$ de los trabajadores son femeninas y el $17 \%$ masculino.

- Al conocer el tipo de empleo en relación a su situación se encontró que 5 personas eran eventuales correspondiente al $16,7 \%$ y el $83.3 \%$ tienen un contrato fijo. De la información recogida se topó que el $56.7 \%$ llevan tiempo laborando en el mismo puesto mientras que el $43.3 \%$ ha experimentado algún cambio.

- En la fábrica se laboran las 8 horas, no existen turnos rotativos, y se considera que las máquinas son seguras.

Según la encuesta los principales riesgos que existen en su actividad se indican en la tabla 5. De los cuales el primer factor de riesgo es la exposición a polvo y pelusas, en este caso es material particulado con un 77\%; luego están los cortes con objetos o materiales debido al uso de elementos de corte como tijeras y cuchillas que se emplean en especial para el corte de hilos; y luego está el atrapamiento con maquinarías.

Tabla 5. Factores de peligro y riesgo

\begin{tabular}{|l|c|c|}
\hline \multicolumn{1}{|c|}{ Factor } & Cantidad & $\%$ \\
\hline Exposición a polvo y pelusa & 23 & $77 \%$ \\
\hline Cortes con objetos o materiales & 19 & $63 \%$ \\
\hline Atrapamientos con maquinaria & 14 & $47 \%$ \\
\hline Golpes con objetos/herramientas & 12 & $40 \%$ \\
\hline Cambios bruscos de temperatura & 8 & $27 \%$ \\
\hline Ruido elevado & 6 & $20 \%$ \\
\hline Lesiones al manipular carga & 6 & $20 \%$ \\
\hline Contacto eléctrico & 4 & $13 \%$ \\
\hline lluminación inadecuada & 1 & $3 \%$ \\
\hline Quemaduras, contacto térmico & 0 & $0 \%$ \\
\hline Caídas de distinto nivel & 0 & $0 \%$ \\
\hline Caídas al mismo nivel & 0 & $0 \%$ \\
\hline
\end{tabular}


Los encuestados consideran que las tareas que realizan pueden ocasionar algún accidente grave, el $47 \%$ responde que a veces, el $33 \%$ indica que no y el $20 \%$ responde que si, como se muestra en la figura 4.

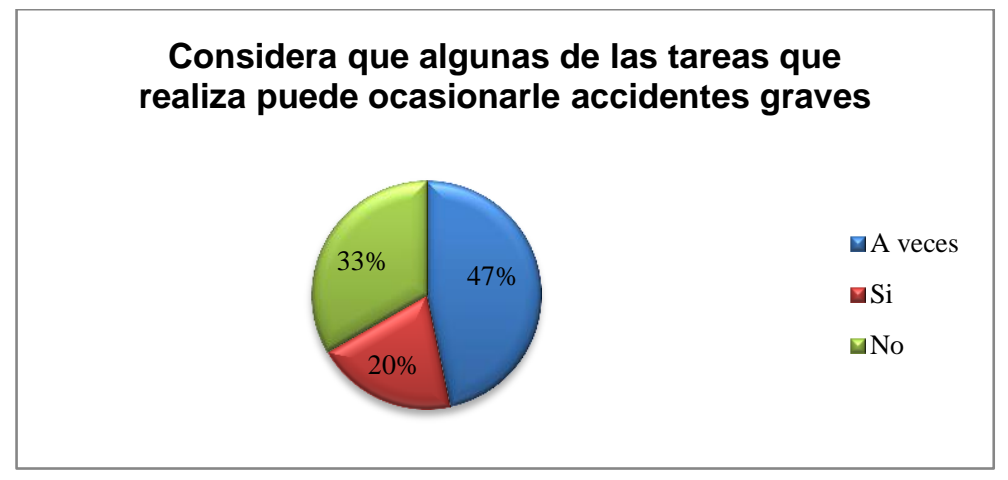

Figura 4. Tareas le puede ocasionar algún accidente grave

De la información recopilada y que se muestra en la figura 5 , sobre si recuerda que se haya producido algún accidente o enfermedad relacionada con el trabajo en la empresa, la mayoría estos indica que no (63\%).

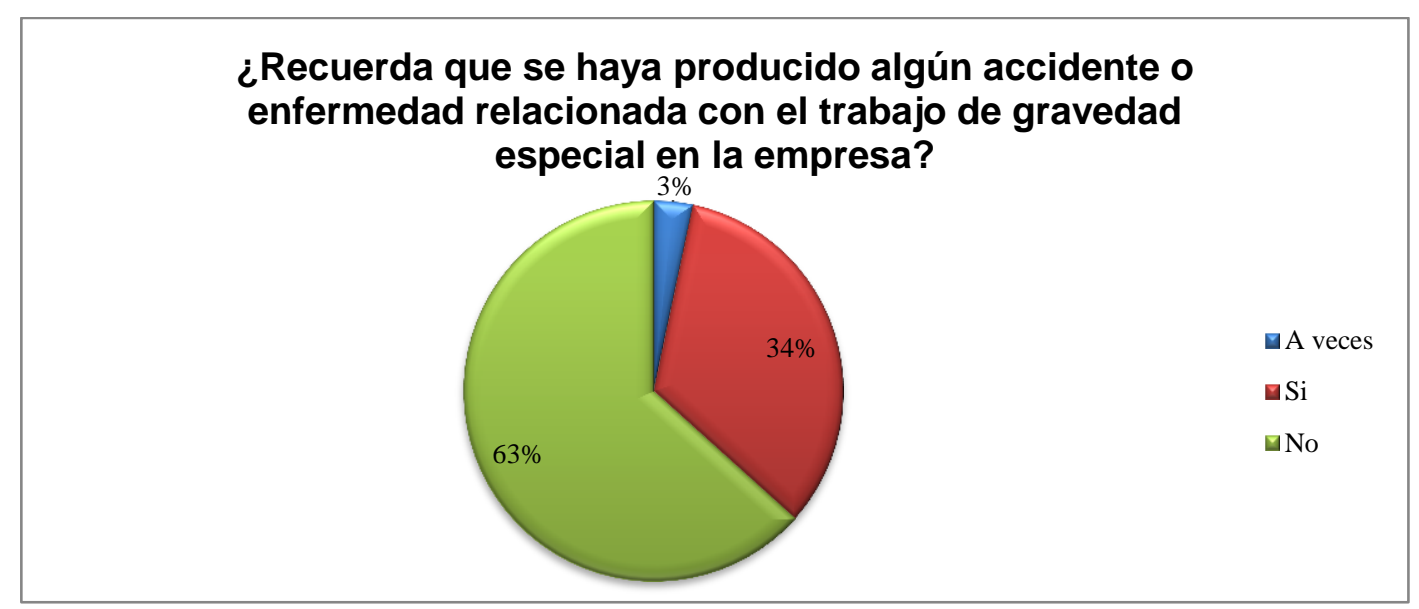

Figura 5. Recuerda algún accidente grave relacionado con el trabajo

La figura 6 muestra los resultados sobre si ha sufrido alguna molestia en el último año relacionada con su trabajo, el $73 \%$ responde que no y el $27 \%$ que si. En este aspecto han sufrido molestias 8 personas de la población encuestada. Estas molestias son en relación al trabajo.

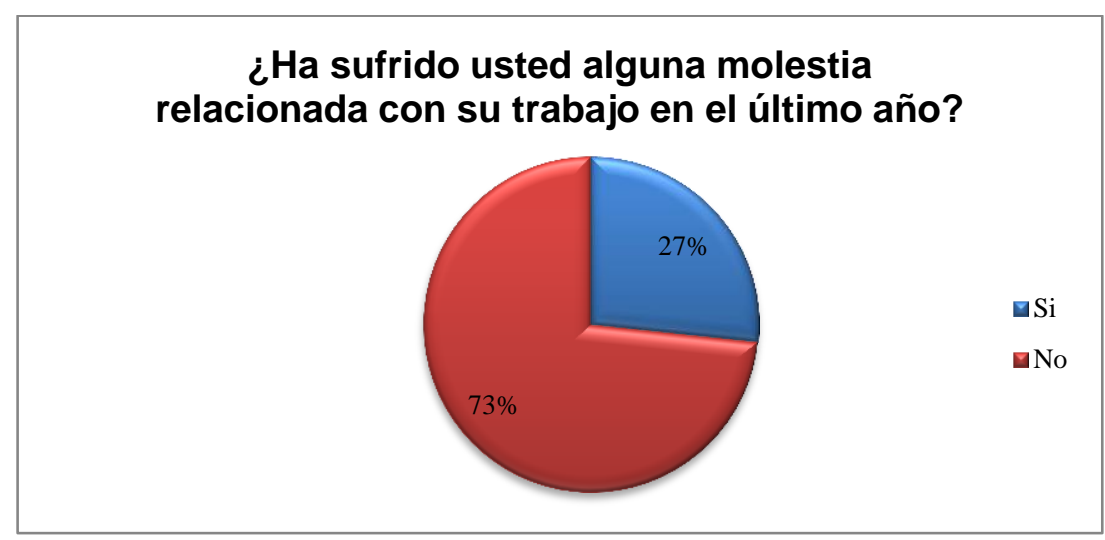

Figura 6. Molestias en el último año relacionadas con el trabajo 
Al preguntar si ha sufrido algún accidente o lesión relacionado con su actividad pero que le haya permitido seguir trabajando, el $63 \%$ indica que no, el $30 \%$ dice que sí y dos personas no responden (figura 7).

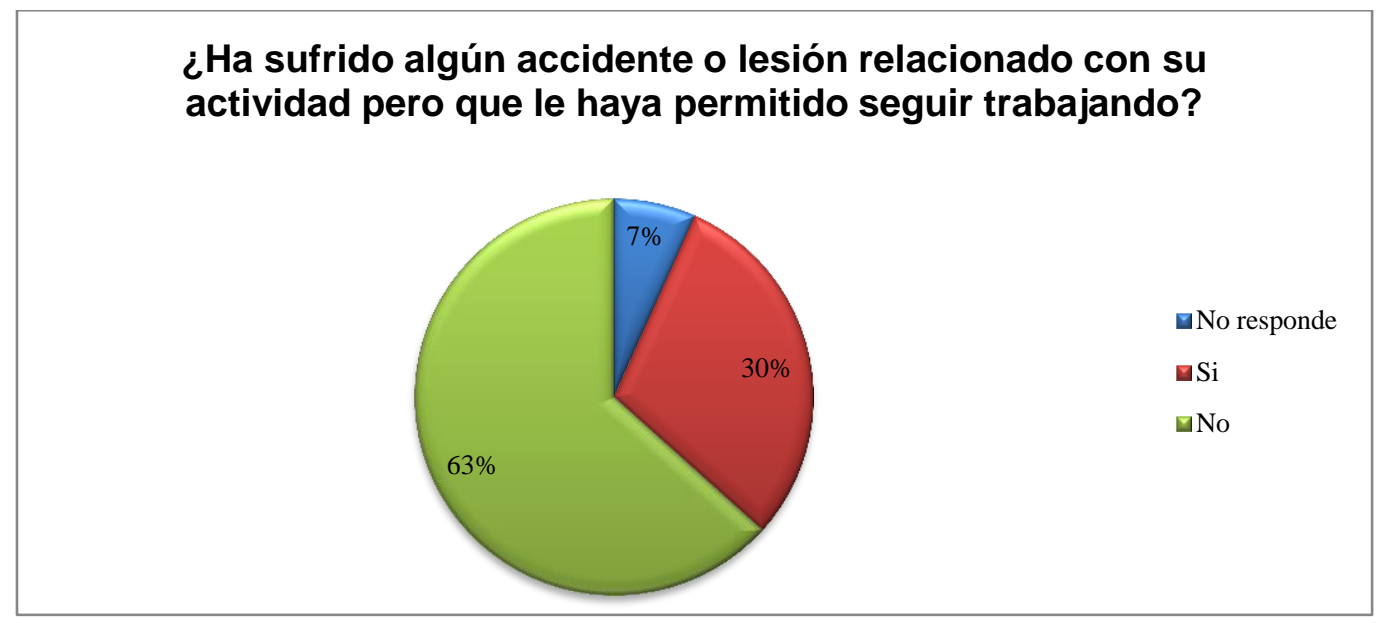

Figura 7. Ha sufrido algún accidente relacionado con el trabajo

Al conocer si ha sufrido algún accidente lesión o problema de salud relacionado con su trabajo a lo largo de su vida laboral, el $73 \%$ responde que no y el $23 \%$ que si (figura 8). En este aspecto apenas 8 personas han sufrido algún problema en relación al trabajo.

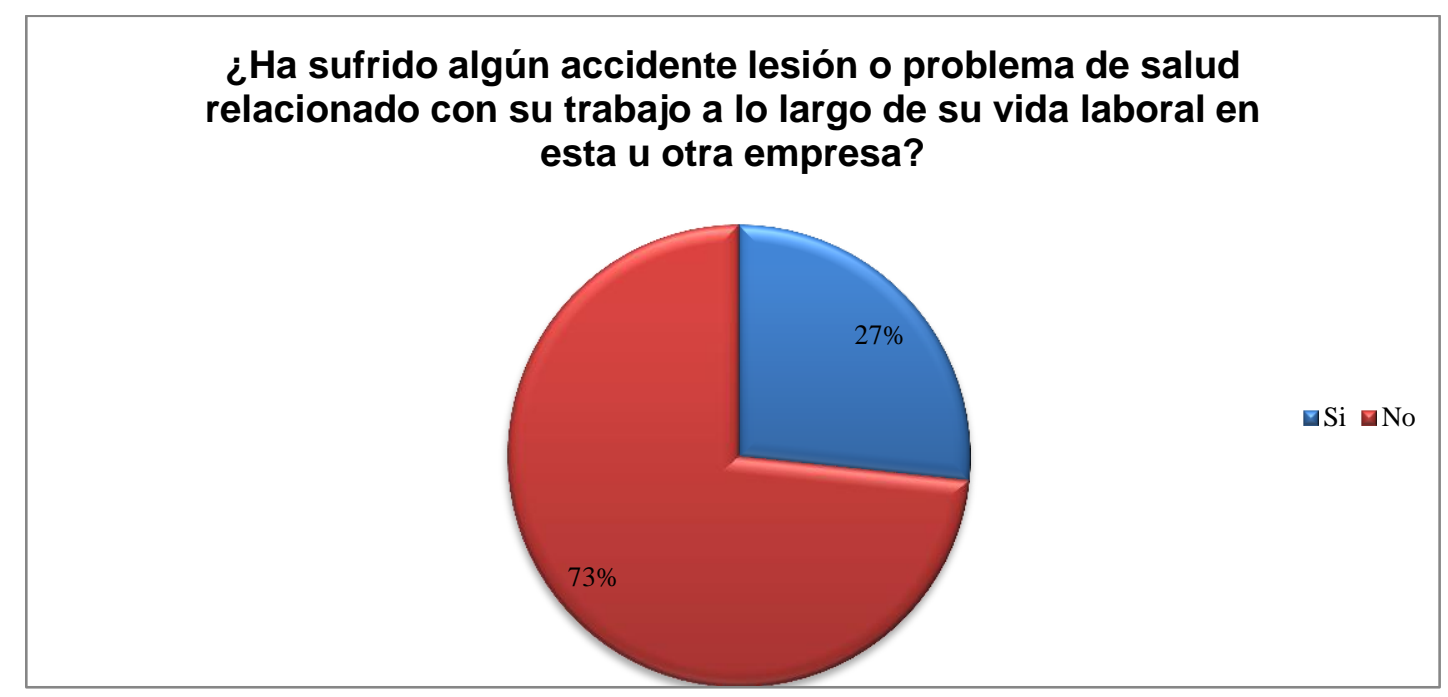

Figura 8. Ha sufrido algún accidente a lo largo de su vida laboral

Cuando se consultó que indique si durante el proceso de su trabajo siente con frecuencia algún síntoma o molestia (figura 9) se observa que la percepción de los operadores es que existe molestias en el sistema respiratorio en un $36,7 \%$ al igual que en sistema visual un $33.3 \%$. 


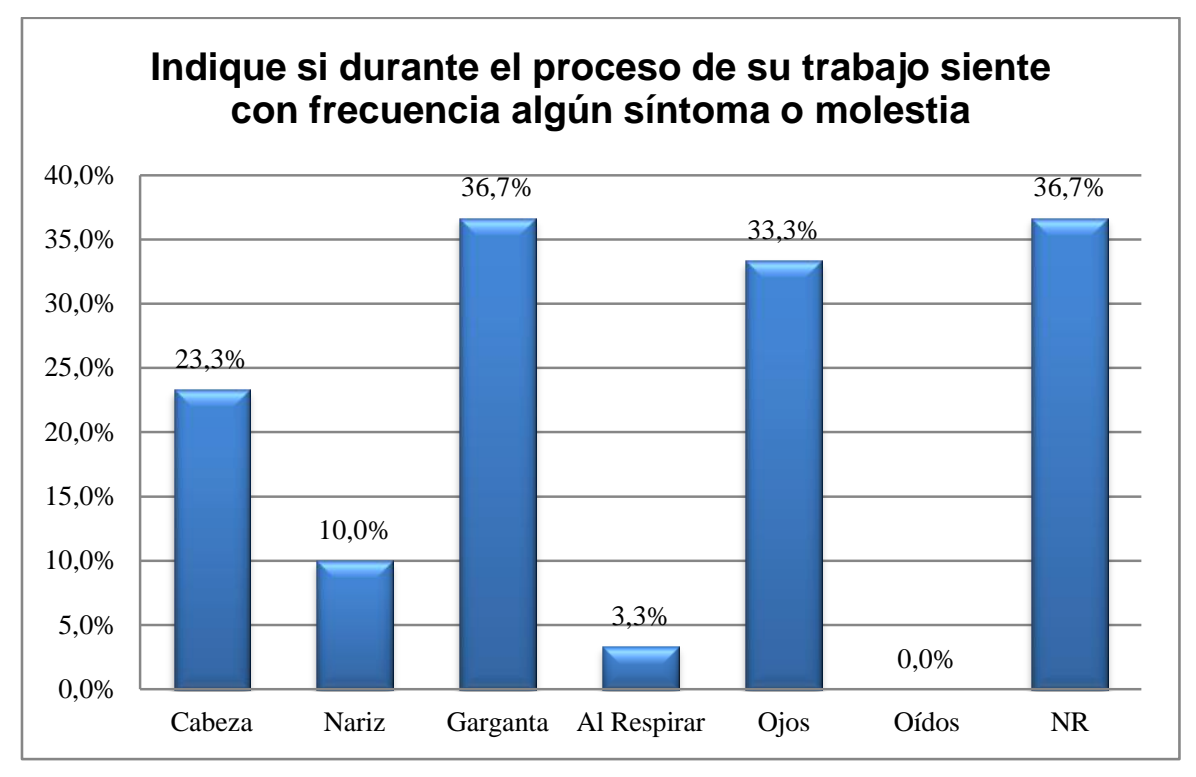

Figura 9. Distribución en cuanto a síntomas

De acuerdo a la opinión de los operadores indica un 83\% que existe compromiso de la dirección de su empresa con la prevención de riesgos laborales (figura 10).

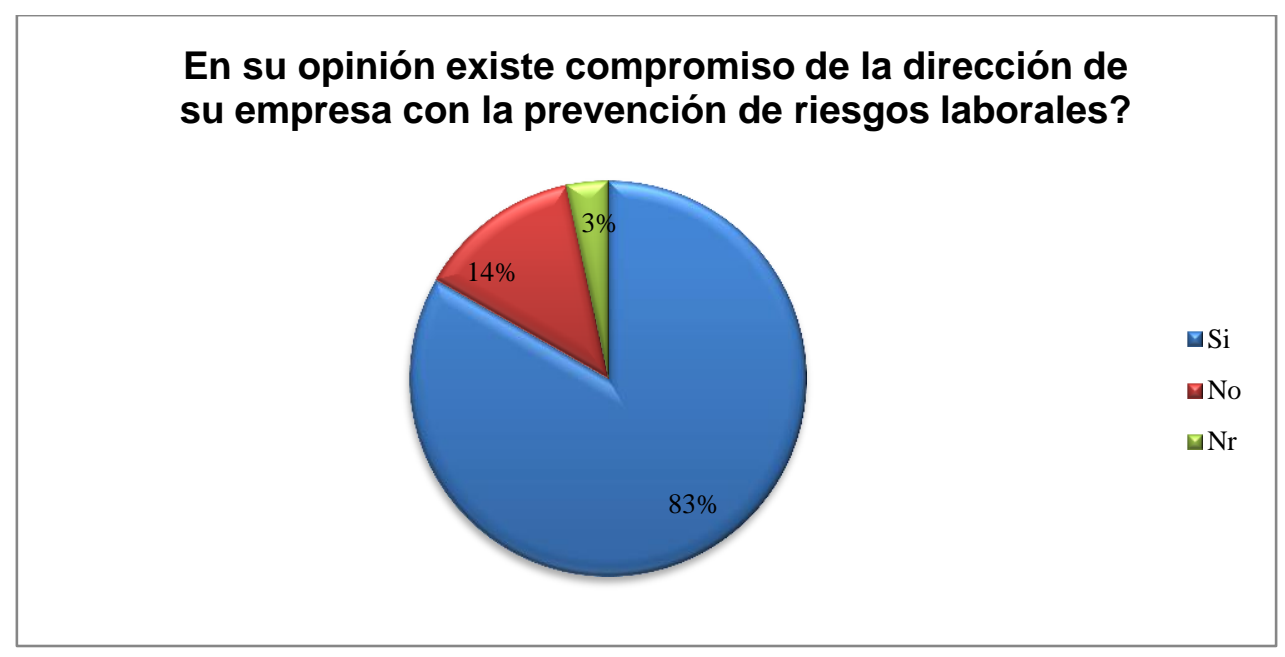

Figura 10. Compromiso de la dirección con la prevención de riesgos laborales

Al preguntar si la empresa les proporciona los equipos de protección personal (EPP's) todos responden que sí, así como todos indican que la empresa si les ha indicado acerca de los riesgos derivados de su actividad laboral y las medidas preventivas para evitarlos.

Al indagar acerca de que si la empresa les ha capacitado sobre los riesgos específicos derivados de su actividad y la manera de cómo prevenirlos una persona responde que no y el resto responde que si han recibido la capacitación constante.

En relación a la información que la organización proporciona al operador para evitar los posibles riesgos en su actividad laboral el $60 \%$ indica que es suficiente, el $40 \%$ restante dice que no (figura 11). 


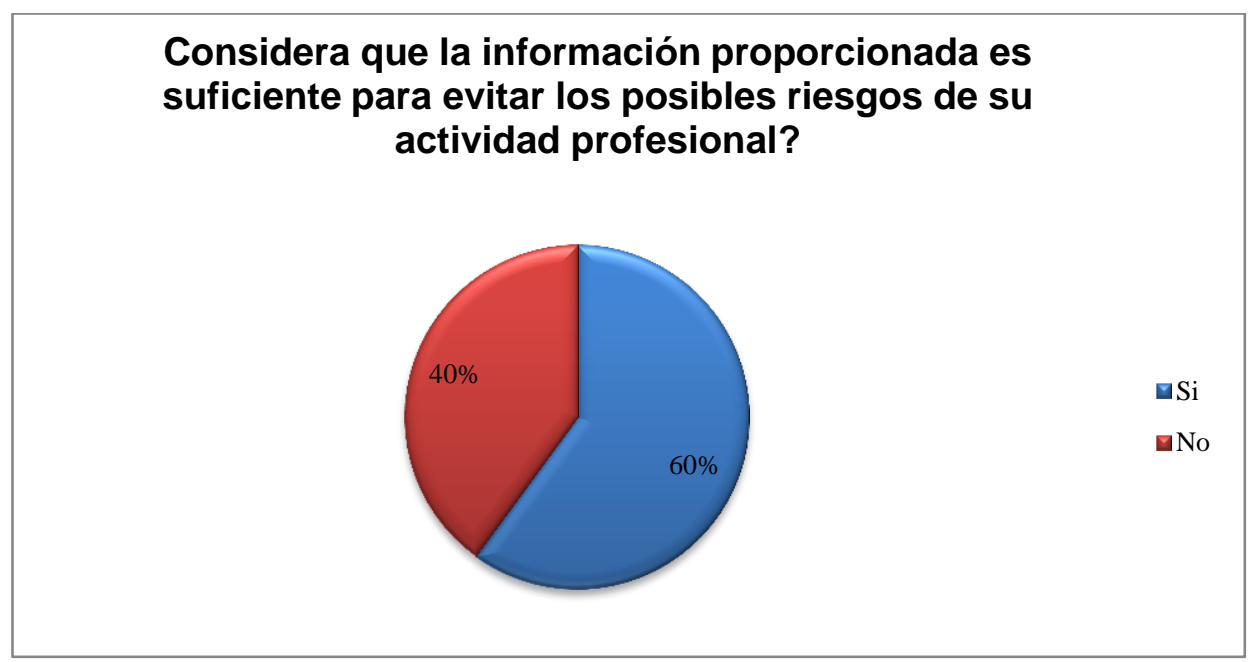

Figura 11. Información proporcionada sobre los riesgos en la actividad.

\section{Conclusiones y Recomendaciones}

\section{Conclusiones}

- De acuerdo a los resultados obtenidos de las mediciones se determinó que existe una variabilidad con el objeto del estudio, los niveles de material particulado son heterogéneos variando de un puesto de trabajo a otro.

- El estudio determinó que en la operación con las máquinas overlock es donde se genera mayor cantidad de material particulado obteniendo un máximo valor en toda la planta de producción de $244 \mu \mathrm{g} / \mathrm{m}^{3}$, y un valor menor en la máquina dobladora con un valor de 9 $\mu \mathrm{g} / \mathrm{m}^{3}$.

- La percepción de los operadores es que existe molestias en el sistema respiratorio en un $36,7 \%$ y en el sistema visual un $33.3 \%$.

- El 73 \% del personal no ha sufrido algún accidente lesión o problema de salud relacionado con su trabajo a lo largo de su vida laboral. 8 personas han sufrido algún problema en relación a la tarea realizada, representando el $23 \%$ de los trabajadores.

- El primer factor de riesgo es la exposición a polvo y pelusa como material particulado con un $77 \%$; luego están los cortes con objetos o materiales debido al uso de elementos de corte como tijeras y cuchillas que se emplean en especial para el corte de hilos; y luego está el atrapamiento con maquinarías.

\section{Recomendaciones}

- Se recomienda realizar evaluaciones ergonómicas de acuerdo a la actividad, pues la misma se efectúa por largos períodos de posición sentada.

- La organización debe mejorar el sistema de información sobre las condiciones de riesgo existentes en la planta. 


\section{Bibliografía}

AITE. (2010). Cifras. Boletin Mensual, 15.

CENELEC. (1995). UNE-EN Atmósfera en el lugar de trabajo. Directrices para la evaluación de la exposición por inhalación de agentes químicos para la comparación de valores límite y estrategia de la medición. Bruselas.

Creus, A., \& Magnosio, J. (2011). Seguridad e higiene en el trabajo. Buenos Aires: Alfaomega.

Gallego C., J., Paeres R., V., \& Gómez, B. L. (2010). Documentación e implementación de un sistema de seguridad industrial y salud ocupacional: una necesidad en las empresas colombianas. USBMed, 1(1), 46-50.

IESS. (1986). Decreto Ejecutivo 2393. Reglamento de Seguridad y Salud de los Trabajadores y mejoramiento del Medio Ambiente de Trabajo. Quito.

INSHT. (2006). NIPO: 211-06-050-8. Toma de muestras de aerosoles. Muestreadores de la fracción inhalable de materia particulada. España.

INSHT Instituto Nacional de Seguridad e Higiene del Trabajo. (2008). Nota técnica de prevención 808. Exposición laboral a agentes químicos: requisitos de los procedimientos de medición. Sevilla, España: INSHT.

Marti Veciana, Antonio; Guardino Solá, Xavier. (2008). NTP 808: Exposición laboral a agentes químicos: requisitos de los procedimientos de medición. Instituto Nacional de Seguridad e Higiene en el Trabajo, España.

OISS. (2009). Estrategia Iberoamericana de Seguridad y Salud del Trabajo 2010-2013. Organización Iberoamericana de Seguridad Social, Estoril, Portugal.

Viteri, J., \& Jácome, M. (2013). La Responsabilidad Social como Modelo de Gestión Empresarial. EIDOS(4), 92-100.

Viteri, J., \& Jácome, M. (2013). La Responsabilidad Social como Modelo de Gestión Empresarial. EIDOS, 92-100. 